\title{
Flat-H Redundant Frangible Joint Design Evolution 2018: Feasibility Study Conclusions
}

\author{
Jacob M. French ${ }^{(1)}$, Dr. Andrew L. Benjamin ${ }^{(2)}$, Christopher W. Brown ${ }^{(3)}$, Thomas E. Diegelman ${ }^{(4)}$, and Todd J. \\ Hinkel $^{(5)}$ \\ ${ }^{(1)}$ Pyrotechnic Systems, EP311, Johnson Space Center, 2101 NASA Parkway, Houston TX 77058, USA, Email: \\ jacob.m.french@nasa.gov \\ ${ }^{(2)}$ Pyrotechnic Systems, EP311, Johnson Space Center, Email: andrew.l.benjamin@nasa.gov \\ (3) Pyrotechnic Systems, EP311, Johnson Space Center, Email: christopher.w.brown@nasa.gov \\ (4) Safety \& Mission Assurance, NE311, Johnson Space Center, Email: thomas.e.diegelman@nasa.gov \\ ${ }^{(5)}$ Pyrotechnic Systems, EP311, Johnson Space Center, Email: todd.hinkel-1@nasa.gov
}

\begin{abstract}
This paper reports results of an investigation into developing a single failure tolerant pyrotechnic linear separation system which features completely redundant explosive trains suitable for human spaceflight. It is a follow up to "Flat-H Redundant Frangible Joint Design Evolution 2017" [1] and "Flat-H Redundant Frangible Joint Evolution" [2]. The paper chronicles the history of the redundant frangible joint development program including testing, analysis, and design improvements from 2014 to the present culminating in a successful proof-of-concept prototype. The paper describes work done to address debris control and containment of combustion products. A performance optimization strategy is presented along with optimization results. Additionally a novel containment manifold design is presented with test results.
\end{abstract}

\section{Intro:}

Human spaceflight has always presented the fundamental challenge of maximizing component reliability and performance in demanding environments while minimizing associated mass, volume, complexity, and cost. Spacecraft designers have historically employed a variety of strategies for preventing component/system failures. These include destructive testing to establish performance limits and failure modes, nondestructive examination to identify faults in components before function, and function testing/cycling of devices during pre-flight checks. Arguably the best strategy for ensuring reliability is fault/failure tolerant components/systems which provide for safe operation in the event that other safeguards fail. NASA's design philosophy has historically called for single fault tolerance for all "must work" critical applications in human-rated spacecraft. Singe Fault Tolerant (SFT) is defined as system performance that allows proper system function with a single failure event occurring. Since the end of the Space Shuttle era, aerospace companies have proposed building human-rated vehicles with off-the-shelf, non-redundant linear separation systems. Current spacecraft and launch vehicle providers for both the Orion and Commercial Crew Programs look to deviate from this conventional approach and from NASA human rating requirements. Commercial partners have baselined zero fault tolerant (ZFT) frangible joints as their baseline for vehicle staging and fairing separation. These off-the-shelf systems can be bought from pyrotechnic vendors and have been flown numerous times for uncrewed missions. These designs however do not feature a redundant independent explosive trains for separation required to be SFT. Beginning in 2008, the pyrotechnics group at NASA's Johnson Space Center began developing a fully redundant single fault tolerant (SFT) frangible joint design suitable as a drop in replacement for baselined ZFT linear separation systems. The design progressed through several initial prototypes reaching a suitable configuration for redundant separation called the redundant frangible joint or RFJ in 2015. The 2015 design, known as pattern ' $G$ ' was prone to over separation and fragmentation at above nominal explosive loads used in margin testing and in post-separation initiation (simulating a hang fire in one explosive train). Limitations in finite element analysis damage predictions combined with operational and financial constraints to testing schedule created difficulty in addressing these concerns. In response, the team began combining existing test data with finite element models to establish useful model performance metrics. These performance metrics were then used iteratively to improve the geometry of the joint in key areas. This process resulted in dramatic improvements in debris/fragmentation control. The RFJ configuration also presented a need for a new initiation and containment configuration for its explosive trains owing to its unique geometry. A new low profile manifold design with an improved sealing interface was developed and tested. The new design yielded exceptional results with complete containment of combustion products with lower mass and volume than existing designs.

\section{Pyrotechnic Separation Systems and Reliability:}

Linear separation systems allow a spacecraft to maintain an optimal configuration through different stages by separating panels and fairings. In any separation system, mass, volume, reliability, pre-separation strength, and 
function time are all critical, but reliability is the paramount concern. Separation failures on uncrewed vehicles have historically led to loss of spacecraft and mission failure. Linear separation systems are often pyrotechnic devices as pyrotechnic devices are generally lighter, smaller, faster, and more reliable than comparable electromechanical systems. Pyrotechnic systems can produce very rapid structural responsesoften within milliseconds of initiation - by transmitting energy in the form of shockwaves. Reliability in pyrotechnic systems, however, demands special attention. By their nature, pyrotechnic devices are single use and cannot be cycled or function tested in operation, as doing so would consume their pyrotechnic charge. Reliability, or fault tolerance, in pyrotechnic devices is instead determined by robust design, manufacturing, and acceptance controls. A key aspect of functional reliability in pyrotechnic system design is redundancy. This is typically achieved by having duplicate energetic trains to function the system. In some cases, entire devices may be duplicated so that multiple independent explosive trains may perform the same task in the event of a single fault. In other cases, redundancy may be designed into a single device by duplicating its pyrotechnic components. In all cases, designs must allow independent explosive trains to achieve the critical function of the device, whether fired separately or in unison. To maintain failure tolerance, these designs must prevent fratricide where the operation of one explosive train causes a fault in the redundant leg. The device must also safeguard against undesired behavior in the event of hang fires where one pyrotechnic train initiates after the desired system function has been completed by another redundant train.

Pyrotechnic linear separation systems for fairing and panel separation are baselined for use in currently planned human spaceflight programs. These systems connect the edges of panels and fairings to one another and to the spacecraft as shown in fig. 1. The joints may be linear running parallel to the central axis of the vehicle or curved around the circumference of the vehicle. This paper will use the following directional conventions: longitudinal/length - along the length of the joint and parallel to the joined panel edges, vertical/height through the panel connection, and horizontal/thickness perpendicular to the length of the joint and the direction of static loading.
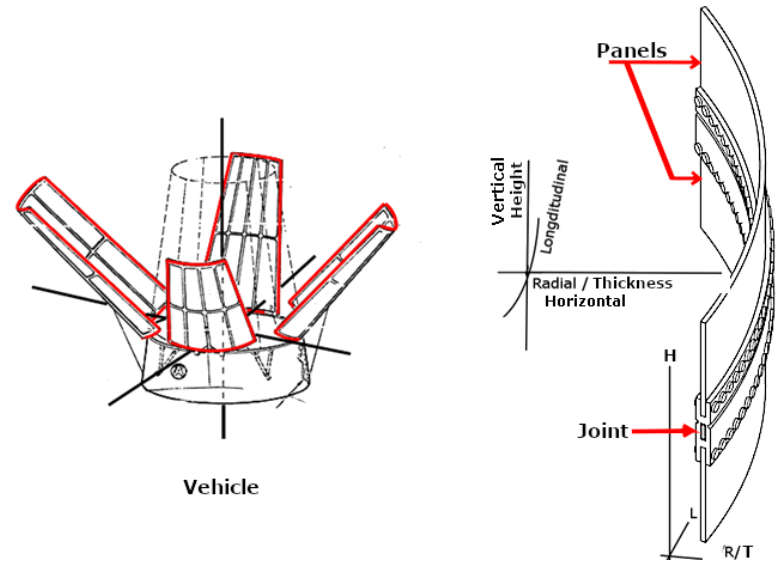

Figure 1: Direction Conventions

When functioned, the joint separates at a predetermined fracture plane in the center of the joint. The fracture plane of the joint is perpendicular to the height direction of the joint with the longitudinal and radial/thickness directions running through the separation plane.

Most commercially available pyrotechnic frangible joint solutions share similar characteristics to accomplish rapid separation and debris control. Separation energy is provided by a length of mild detonating fuse (MDF) containing a high explosive core that is surrounded by a soft metal sheath. This cord is contained in a flattened steel tube and held in place by an elastomer charge holder. Together, the cord, charge holder, and tube make up the expanding tube assembly or XTA, which is packaged inside a set of aluminum plates or clevis, which are notched at the desired fracture plane. The section of the joint in the fracture plane is called the ligament. This arrangement appears in fig. 2

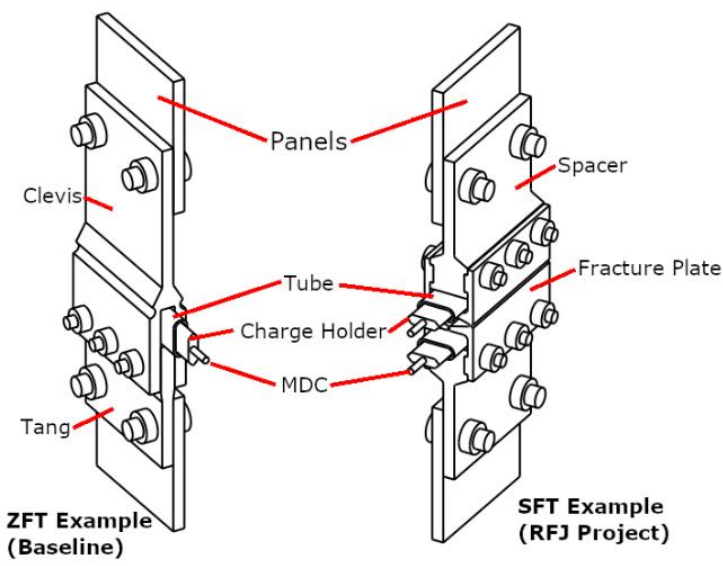

Figure 2: SFT and RFJ Configuration

When the cord is detonated, it supplies pyroshock and pressure to the XTA. The charge holder material helps transfer these forces to the tube. The combined forces on the tube cause it to expand and impact the plates of the joint. The impact results in a concentrated shearing stress in the ligament of the joint. When this stress exceeds the 
material strength of the ligament, the joint fails rapidly along the fracture plane. The functional by-products of the MDF are contained in the steel tube, preventing the release of debris. The separation process is very rapid. Detonation progresses along the longitude of the joint at several thousand meters per second and complete separation at any cross section is achieved within microseconds of the detonation reaching that location. The entire process, from initiation of the MDF to complete separation of the joint, lasts only a few milliseconds.

Frangible joint designs like the tang clevis arrangement shown in fig. 2 have only a single XTA making them zero fault tolerant (ZFT.) To comply with NASA's historic SFT design philosophy, a frangible joint design for human rated critical applications will need two redundant explosive trains each capable of achieving separation by themselves. This need was reaffirmed in 2017 when the National Engineering \& Safety Center (NESC) and NASA Safety and Mission Assurance (S\&MA) both concluded that development of a redundant frangible joint is a priority for future human spaceflight efforts. [3]

\section{Adapting Separation Systems to Address Redundancy}

At its earliest stages, the RFJ team developed design concepts based on the requirements of the Altair Lunar Lander project. These concepts were similar to Tang Clevis joints with vertical XTA's above and below the fracture plane intended to shear the joint at a common fracture plane. Analysis of these joints showed they would be susceptible to buckling under high compressive loads. To reduce buckling under compression loading, the Flat-H RFJ concept was developed. The Flat-H pattern places the joint tubes in a horizontal direction, thereby increasing joint stability and reducing the height of the joint when compared to earlier dual tube concept designs. The horizontal arrangement of the XTA's changes the separation function of the joint slightly. The Flat-H design fractures the ligaments by bending them as the XTA expands; ZFT tang-clevis style joints primarily place the ligaments in shear stress. The operation of the two styles is shown below in fig. 3 for comparison.

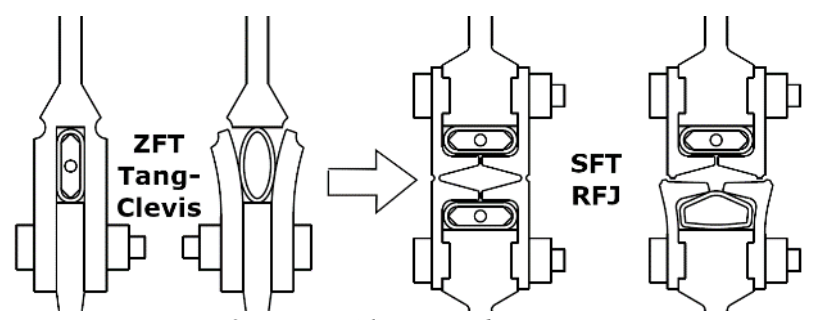

Figure 3: Tang Clevis and RFJ Function

In 2013, the Flat-H concept was selected for further development for its stability and for having comparatively low volume and axial length compared to other concepts.
The RFJ design has several advantages over the tang clevis style joint. The RFJ is inherently redundant with two separate independent explosive trains. The RFJ is also more resistant to buckling failure under compressive load due to its greater width in the short transverse/radial direction. The RFJ is not without its drawbacks however. The addition of a second XTA and added structure is a significant weight penalty, around 1.5 times the mass of a typical tang-clevis design. The change in ligament fracture from pure shear to bending adds some complexity to separation under load. The arrangement of the two XTA's close to one another precludes the use of existing end containment manifolds due to their size. Finally the bending in the hinges required for separation of the joint often resulted in damage to or failure of the hinges during final expansion of the XTA after separation was complete. These final two issues, secondary fracture and end containment, were major development hurdles. Release of debris or fragmentation would present a major concern for any spaceflight applications.

\section{Hinge Fragmentation:}

In 2015 changes were made to the interface between the fracture plates and spacers of the redundant frangible joint to improve energy transmission from the XTA to the fracture plates. The updated fracture plate design was designated 'concept $G$ ' (this paper will refer to it and other variants by their alphabetic pattern designation). The pattern ' $G$ ' fracture plates had a keyway feature added which fit into a recessed notch in the spacer plate. This design change prevented slippage between the spacer and plates which had previously reduced energy transmission between the plate and spacer. The change has the intended effect of improving energy transmission allowing separation at lower load than was previously achieved. The improved energy transmission however exposed a new issue. After separation of the joint, the continued expansion of the XTA caused the hinges of the joint to crack at the root of the radius between the arm and hinge. An example of this cracking appears in fig. 4.

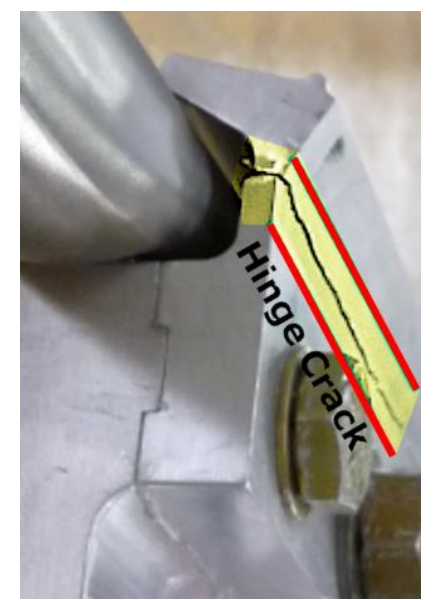

Figure 4: Hinge Cracking in 'G' Pattern Joint 
This cracking posed a serious issue, especially in postseparation testing where the hinges would often fail completely and create dangerous flying debris. This issue will be referred to as 'secondary fracture' or 'fragmentation.' Fragmentation had not been previously observed in RFJ testing, presumably due to low energy transfer from XTA to the joint. The FEA models being used to analyze the RFJ designs did not predict failure or damage in the observed region. Figure 5 shows a detail of the fracture plate with important regions highlighted and numbered.

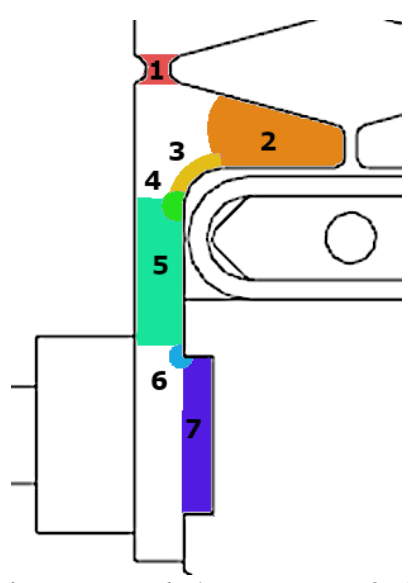

Figure 5: Flat H Detail. 1-Ligament, 2-Arm, 3-ArmHinge Fillet, 4-Arm-Hinge Fillet Root, 5-Hinge, 6-Key Root, 7-Keyway

\section{Stopgap Solution:}

The team began investigating hinge fragmentation with two initiatives, adding structural features to the RFJ to mitigate secondary fracture, and investigating the FEA model to understand and improve modeling of damage in the hinge region. In order to leverage existing test assets, the team implemented 'retainer plates' as a stopgap solution. This appears in fig 6 .

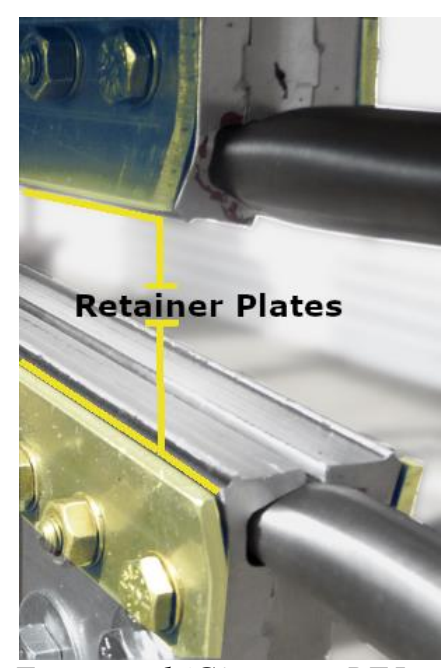

Figure 6: Functioned ' $G$ ' pattern RFJ with retainer plates
These plates bolted to the outside of the RFJ arrested expansion of the joint after separation. Testing showed these retainer plates were effective in preventing damage in the hinge region of the joint without negatively affecting the separation characteristics of the joint. This was an important result as it demonstrated that the damage and failure of the hinges began after complete separation of the joint rather than during the ligament fracture. The joint had separated completely before contacting the retainer plates, but the hinge cracking had not yet begun. This would later be confirmed by high framerate photography of joint separation and hinge fracture.

Though effective at checking overexpansion, the stopgap solution using large retainer plates had two major drawbacks. The plates are heavy and bulky significantly increasing mass and volume of the joint. The space between the retainer and fracture plate is also a risk for collecting FOD or ice buildup which, if trapped between the retainer and fracture plate, could potentially cause a failure to separate. These two issues led the team to pursue a redesign of the joint geometry which could address overexpansion/secondary fragmentation without the external retainer plates required to make the ' $G$ ' pattern work.

\section{Hinge profile Modification:}

The first redesign attempt to address secondary fracture was made in 2016. It was decided to approach the problem by thickening the hinge in the area of observed failure to reduce strain. Material could not be added to the inside of the radius, so the outside of the hinge was thickened near the arm-hinge radius, and near the hinge root where stress concentrations could be expected. The hinge remained narrow in the center, preserving its ability to bend and produce the desirable rotation at the ligament necessary for joint separation. The new design, pattern 'I' was otherwise similar to the previous pattern ' $G$ ' geometry. Existing FEA models were used to analyze the new design for separation characteristics with positive results but as previously noted the FEA model did not provide useful predictions for hinge damage and failure. Figure 7 shows the updated I pattern geometry next to the ' $G$ ' pattern geometry where hinge fragmentation was first observed. 


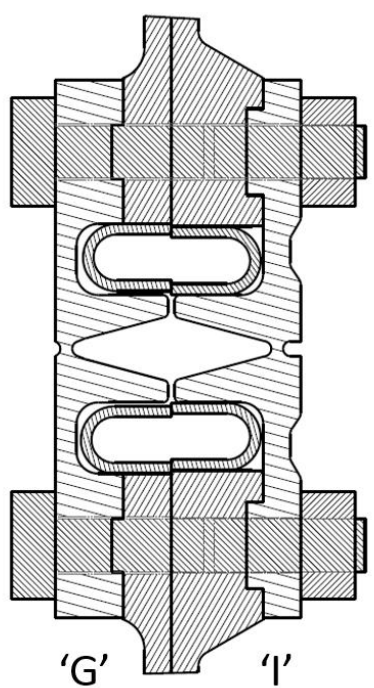

Figure 7: Patten G and Pattern I comparison

The new geometry 'I' was tested extensively in 2016 including evaluating separation at the new lower MDF coreload in previously marginal test conditions, determining static loading capacity in tension and compression, and attempting to diagnose the newly observed secondary fracture issues. The 'I' pattern geometry reliably separated with a single, nominal coreload XTA, but damage and failure of the hinges was seen in every test. Static load testing indicated the design significantly exceeded static load capacity requirements. The joint supported more than $300 \%$ of required load in tension and compression. This excess static strength indicated that a reduction in ligament sizing accompanied by a similar reduction in explosive loading could be a viable path to eliminating hinge damage. This was not pursued due to limited availability of different size MDF. High framerate photography of the 'I' pattern gave additional insight into the hinge damage mechanics. The damage generally initiated just as joint separation completed and proceeded across the hinge as the joint continued its expansion. Photon Doppler Velocimetry (PDV) and Digital Image Correlation (DIC) data provided additional model feedback for tuning separation times in the model to displacements and velocities. Investigation was also done into PDV traces to look for velocity and position data that might be used to predict future margin in hinge performance. This PDV data was critical to improving and validating FEA models. Figure 8 shows a comparison of Model and Test data for the positions and velocities of an 'I' pattern joint above and below the hinge.
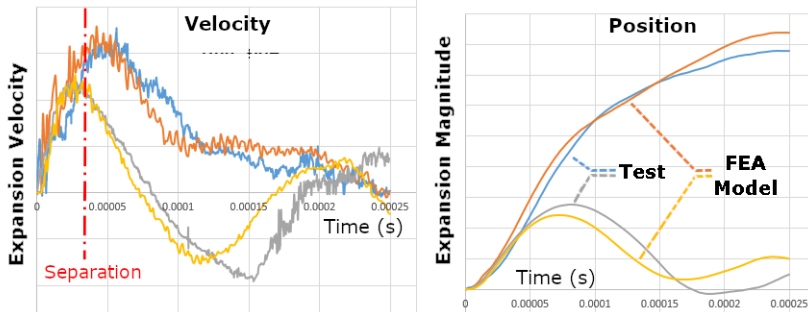

Figure 8. Comparison of Model and Test Data, Pattern ' $I$ '

\section{Low Profile Retainer Evaluation}

After evaluating the negative effect of the 'I' pattern hinge profile modifications, the RFJ team returned to the straight sided hinges of the ' $\mathrm{H}$ ' pattern joint which had displayed fragmentation control with large retainers. In order to reduce the mass penalty and trapped FOD risks associated with the previous retainer configuration, new low profile retainers were added. These retainers were thin aluminum plates sitting flush with the outside of the hinge. The intent was for the retainers to stiffen the outside of the joint reducing expansion by absorbing energy as they deformed. FEA of these low profile retainers suggested minimal effect on joint function, so the team sought to test 3 articles for comparison, the old 'I' Pattern joint, the new ' $J$ ' pattern joint with low profile retainers, and a ' $\mathrm{J} 125$ " joint a $25 \%$ increase in hinge thickness in addition to the retainers. Figure 9 shows the 'I'. 'J', and 'J125' patterns.

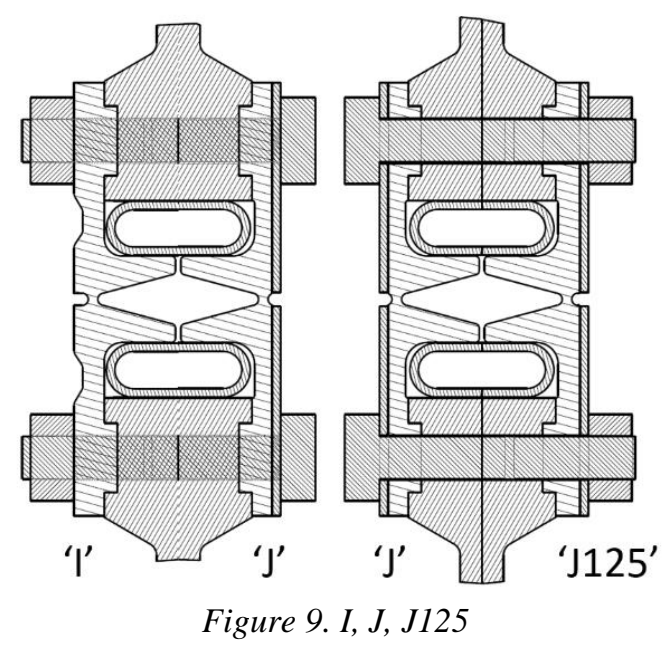

In addition to the linear ' $\mathrm{I}$ ' and ' $\mathrm{J}$ ' articles, a curved ' $\mathrm{I}$ ' pattern subscale section was evaluated. This curved subscale was built to demonstrate manufacturability of the new RFJ configuration in circumferential applications. Test results were mixed. Though the ' $J$ ' pattern joints still separated reliably, hinge damage and failure still occurred at $110 \%$ nominal coreload. The 'J125' joint with thicker hinges was a minor improvement, hinge damage still occurred but the hinges did not completely separate from the joint. The low 
profile retainers were not effective at mitigating hinge damage. Finally the curved subscale of the 'I' pattern joint demonstrated reliable separation. The curved I pattern also exhibited partial hinge failure with the inside hinge failing, but the outside hinge exhibiting no damage. Hinge failure was expected in the curved ' $I$ ' subscale based on previous testing. Long manufacturing times for the curved subscale necessitated choosing an existing geometry rather than delaying until hinge damage could be resolved.

\section{Early Optimization Work - 'K' Pattern}

Testing of the 'I' and ' $\mathrm{J}$ ' pattern joints exposed significant deficiencies in the FEA models. Though effective at predicting ligament separation, the models failed to predict hinge damage. In late 2017 the team adopted a new approach. The pattern 'J' would be used as a baseline to optimize the geometry of existing features while investigating FEA model improvements to better predict hinge damage. To optimize the joint design the team sought to identify geometry changes which could improve separation performance while mitigating hinge damage.

The team began by establishing a simple 2-D FEA model which could be used to quickly make and compare changes to the behavior of the hinge and ligament areas of the joint. New 2-D models allowed quick run times to evaluate numerous small geometry adjustments quickly. The 2-D model was then used to determine which performance characteristics were impacted by small geometry changes and which were constant. The results were quite striking. Peak stresses in the hinge and ligament were similar across a broad range of geometric changes, but peak strains in the hinge at the root of the arm-hinge radius varied greatly. In many cases minor geometry changes pushed peak strains well past the low strain rate elongation expected for failure though the Johnson-Cook material model used did not predict damage in the region. The second major finding was the broad range of function times for complete separation at the ligament. Based on these results, the team decided to begin optimization work to minimize both peak hinge strain, and time to complete ligament separation. A broad variety of geometric parameters were optimized, and major changes included moving the ligaments outward, increasing the hinge radius, and adding a chamfer to the tip of the fracture plate arms. Ultimately, these changes gave significant predicted performance improvements. Predicted peak strain was reduced by $\sim 30 \%$ while predicted function time was reduced by almost $50 \%$. Fig. 10 illustrates the improvements by iteration stages.

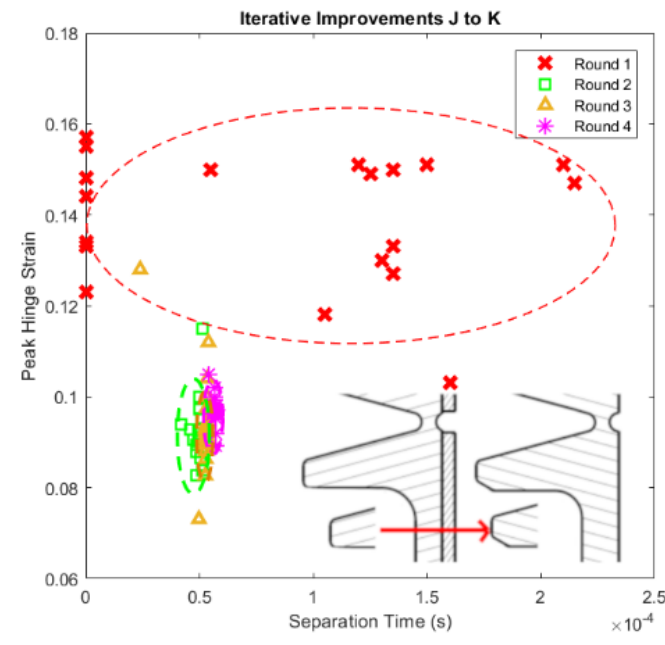

Figure 10. Performance Improvements $J-K$ (Markers with Sep time of 0 failed to separate.)

This optimization established the design for the ' $\mathrm{K}$ ' pattern geometry seen in fig. 11 .

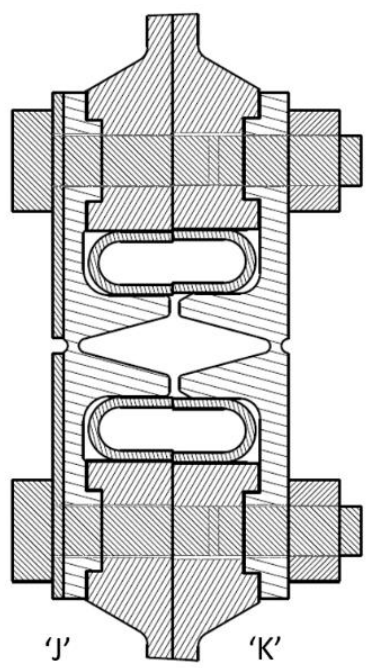

Figure 11. Comparison of baseline ' $J$ ' and optimized ' $K$ ' pattern geometry.

Since the model predicted significant positive margin on separation with the ' $\mathrm{K}$ ' pattern it was decided to test with and without introducing a small $(<0.01$ ") standoff gap between tube and arms. FEA models predicted a significant reduction in peak strain with the standoff gap. The inside surfaces of the hinges were also polished to eliminate 'machining steps,' small surface discontinuities from the machining process which act to concentrate stress at a particular point in the hinge. This too was supported by FEA where small discontinuities introduced in the hinge radius led to dramatic localized deformation or cracking. Finally a thin reinforcement plate was added between the washers and side of the fracture plates to better distribute the bolt preload forces and reduce deformation near the 'Key roots' where the 
base of the hinge extends out to interlock with the spacer This location was highlighted in Fig. 5 location 6.

Testing of the ' $\mathrm{K}$ ' pattern joints was performed in June 2018. The ' $\mathrm{K}$ ' pattern testing used higher explosive loads, $110 \%$ of nominal coreload, as the focus was eliminating fragmentation in post separation firing at high coreload. Separation with and without retainers was achieved as expected, in both cases some hinge damage was observed but significantly less than was previously observed in the 'I' and ' $\mathrm{J}$ ' patterns with shims. Post separation firings were likewise a noticeable improvement over previous iterations. Retention of the containment tube in post separation was achieved for the first time with the ' $\mathrm{K}$ ' pattern joint. These test results marked a major performance milestone for the program - fragmentation control at $110 \%$ nominal coreload, and provided validation for the hinge strain optimization approach employed. A tear down of the ' $K$ ' pattern articles showed damage initiation in the key areas of the hinge, an area where damage had not previously been observed. This damage is highlighted in fig. 12.

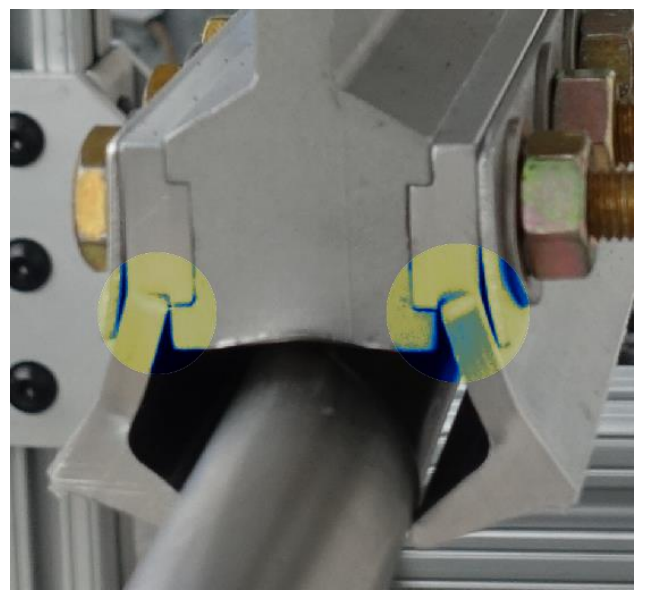

Figure 12. 'K' pattern Key Root Cracking (Highlighted)

This indicated that increased strain in the key root would need to be addressed in the next iteration along with further reduction in the arm-hinge root.

\section{Design Updates and Further Optimization - ' $L$ ' and ' $M$ ' Patterns}

' $\mathrm{K}$ ' pattern testing demonstrated the effectiveness of the optimization strategy but improvement was still required. Successful fragmentation control in post separation firings with low profile retainers still showed some damage to the hinges. Stress concentrations in the key root showed crack initiation. The team decided to further optimize the joint with the goal of eliminating all hinge damage while using shims to prevent any gaps or standoffs in the joint configuration. To achieve this the team investigated the addition of three features to the geometry. The first feature was a variable radius hinge to better distribute the deformation strain of the hinge along the fillet. The second feature was an extrusion to the outside of the base of the hinge. This took the place of the low profile stiffening retainer from the ' $\mathrm{K}$ ' pattern geometry and was intended to reduce strain at the key root. The final change examined was a radius in the corner of the key root. The ' $L$ ' pattern geometry was given nominal dimensions for the first two of these added parameters, but did not feature a radius at the key root. Otherwise it was similar to the ' $\mathrm{K}$ ' pattern which was previously optimized and tested. An ' $M$ ' pattern joint was designed by optimizing the geometry of the ' $L$ ' pattern joint with particular focus on the new geometric features. The ' $M$ ' pattern joint was also given a radius at the 'key root.' Figure 13 shows the 'L' and 'M' pattern geometry.
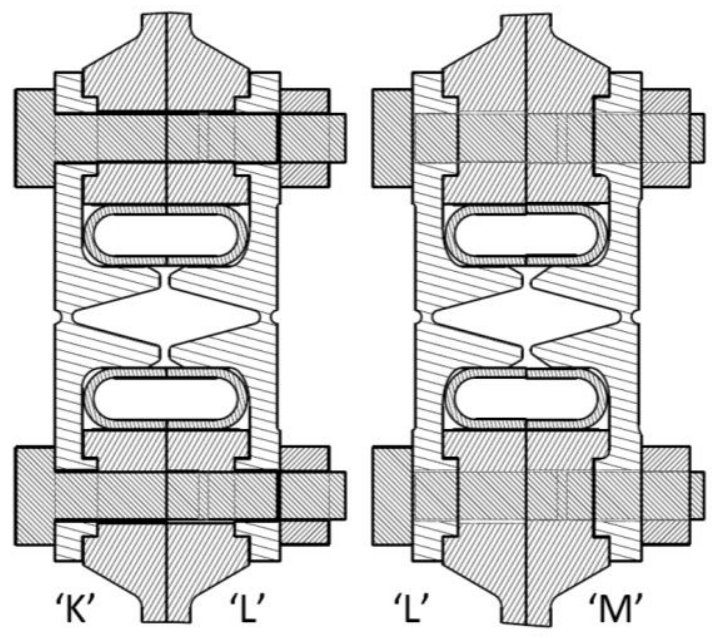

Figure 13. K, L, and M Patterns

To optimize the new ' $M$ ' pattern geometry separation time and hinge strain were again minimized. Greater focus was placed on distributing strain evenly rather than simply minimizing peak strain. To facilitate this, the predicted peak strains along the inside of the hinge for each design iteration were plotted and compared. This gave a clear visual comparison of stress distribution over the surface of the hinge. This slight change to the previous optimization strategy was a significant improvement. It allowed the team to rapidly compare the effects of minor geometry changes across the entire hinge profile of the joint at once. This allowed a design which maximized expansion while reducing the likelihood of hinge damage by evenly spreading deformation. Figure 14 shows the evolution in 'hinge strain profiles' from FEA models for the designs discussed in this paper. 


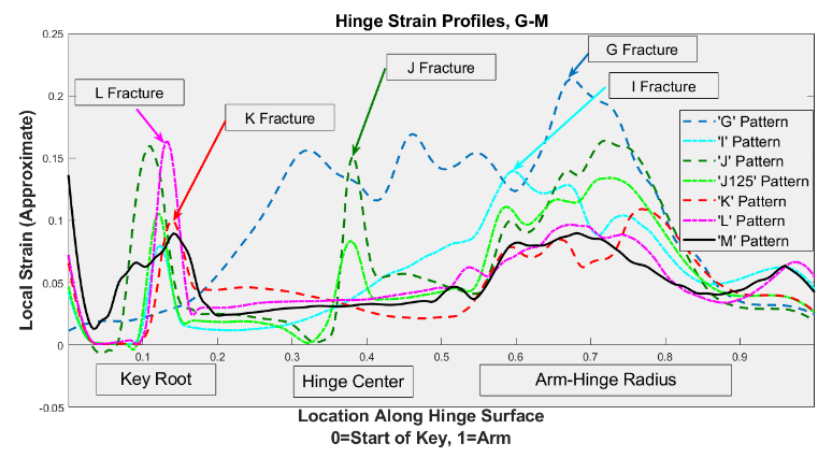

Figure 14. Hinge Strain Profiles, patterns G through M

In addition to geometry changes, the team added a short section of retainer to the first two inches of each end of the joint. The idea behind this was to support the free ends of the joint where the expansion and strain were expected to be highest based on previous tests.

Testing of the ' $L$ ' and ' $M$ ' plates was performed in July 2018 and was again done with 30GPF coreload to evaluate hinge damage in nominal and post separation conditions. The ' $\mathrm{L}$ ' plate showed improvements in the arm-hinge fillet root, but still failed in the key root in post separation firing. The optimized ' $M$ ' plates were fully successful with no damage to the plates, even in the post separation firing. The introduction of the radius at the key root had alleviated the crack initiation at the key root and the optimized joint showed no indication of damage at the hinge. This major milestone marked the first fully successful post-separation firing of the RFJ.

\section{Containment Manifold Work}

In addition to hinge damage/secondary fragmentation, the RFJ design presented a new challenge for initiating the explosive charge within the XTA and sealing the resulting combustion products. The RFJ design has two XTA's positioned with their flat sides near to each other. Existing manifold designs were too large to fit on the ends of RFJ XTA's without interfering with one another. Industry designs generally featured a manifold body with a port for attaching an FCDC connected to a grooved plug extending into the center of the XTA. Clamps were then applied to the outside of the XTA to crimp the XTA onto the manifold plug. This design worked well to secure then manifold to the XTA and transfer the explosive train from FCDC to XTA, but were bulky and often allowed some blow-by of hot combustion products.

To address dimensional and blow-by challenges the team added a recessed groove to the XTA-Manifold interface. The XTA slots into this groove in assembly and during function the groove supports the exterior of the XTA. In this configuration the internal pressure within the XTA supports the seal interface rather than working against it. Securing the Manifold to the XTA was still accomplished by crimping the XTA to an internal plug. This configuration, the XTA Pressure-Sealing Containment
Manifold (XPCM) or horizontal manifold assembly (HMA) is shown in fig. 15.
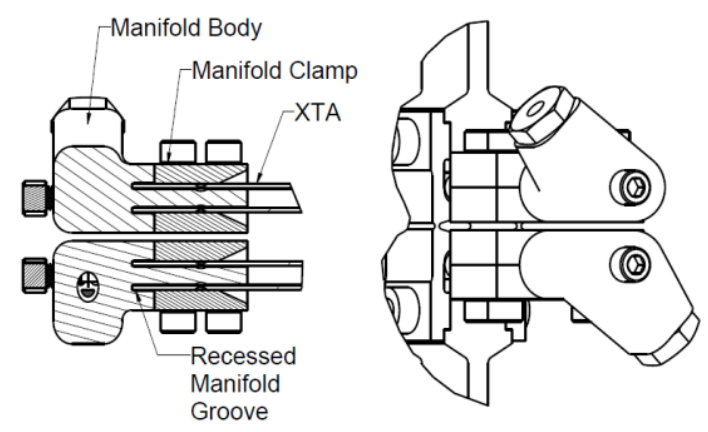

Figure 15. RFJ XPCM Horizontal Manifold Assembly

The combination of the internal XTA plug and recessed groove presented a significant manufacturing challenge as the groove was deep, narrow in width, and required reaching past the 1 " long plug to machine. Machining this groove conventionally was deemed unfeasible due to the high cutting force, small diameter, and long reach it would require. Instead the team perused two alternate approaches; brazing and direct metal laser sintering (DMLS). The brazed manifold strategy used a two part construction with the outer collar being brazed to the inner core and plug. This provided the desired geometry in a conventionally machined package, but required tight tolerances ( 0.001 ") to achieve a good brazed joint. The second method, DMLS, was simpler allowing direct construction of the required geometry by additive manufacturing.

The first round of testing on the new manifold design was performed in April 2018 using XTAs placed inside RFJ test articles which had previously failed to separate due to a manufacturing error. Test results were promising, but sharp edges on the manifold clamps resulted in the tube rupturing at the clamp-XTA interface. Teardown and sectioning of the manifold specimens showed no evidence of blow-by through the XTA manifold interface. Additionally the tests demonstrated the advantages of redundant configuration by separating after failure of the first XTA.

To eliminate tube rupture on clamp edges, new sets of 4 prototype clamp designs were developed and tested. All four clamp designs worked satisfactorily in allowing the XTA to operate without rupture or damage.

Results from the first round of testing, and the clamp development were incorporated into a second set of manifold prototypes. These prototypes were all manufactured by DMLS and were again incorporated into RFJ designs which had previously demonstrated marginal separation performance. Single and dual fire testing was performed in September 2018 to demonstrate initiation of the explosive train with complete containment of combustion products. Testing was fully successful in both configurations. High speed video and 
post-test teardown and inspection of the manifolds showed no evidence of blow-by or of interference between the two XTA's.

\section{Current State of the RFJ Project}

Elimination of secondary fragmentation, and explosive transfer/containment were the final two major obstacles addressed by the RFJ feasibility study. With successful testing of the optimized ' $M$ ' pattern joints, and the RFJ compatible containment manifolds complete, the project has confirmed a reliable redundant frangible joint configuration is feasible. The demonstrated configuration is capable of exceeding requirements for static loading in tension and compression. The RFJ separates cleanly without fragmentation in simultaneous, staggered, and hang-fire simulations. It can be produced and functioned in linear and curved subscale sections. It has a demonstrated solution for initiation and control of combustion products. The team has demonstrated an effective approach to tuning joint response with FEA models. These major milestones have significantly raised the technical maturity of the project. The RFJ has demonstrated a successful redundant configuration for frangible joints.

\section{Potential Future Work}

With debris control and XTA containment demonstrated, forward work should identify functional margins for the RFJ design and continue optimization. First, testing of functional margins should include explosive coreload minimums and maximums. Second, performance under compression loading has should be demonstrated in evaluated with high fidelity FEA modeling and testing. This performance margin is important to establish for implications in scenarios like an abort where separation might be desired under sudden acceleration of the vehicle. Third, further work should be done to measure and manage pyro-shock in operation. It is currently unknown how pyro-shock from the RFJ design compares to existing ZFT frangible joints. Mitigation of pyro-shock might also work with further optimization of the RFJ to perform at lower explosive loads. Finally optimization of the joint design may consider reduction of the delta mass penalty of the SFT RFJ compared with ZFT joints.

\section{Conclusion}

Successful testing of the ' $M$ ' pattern RFJ and XPCM horizontal containment manifolds were the final milestones in the Flat-H Redundant Frangible Joint Feasibility Study. The study has demonstrated a working prototype for a fully redundant SFT frangible joint configuration suitable for current human spaceflight programs. The design of the " $\mathrm{m}$ " pattern joint is by no means final. As this study has demonstrated, optimization of the joint allows for significant 'fine tuning' of response, and much work is still to be done in optimizing for mass, functional margin, shock transmission, loading, and other important factors. The results of the feasibility study suggest that these challenges can be addressed with confidence in the underlying configuration.

\section{References}

1. (French, Hinkel, Brown, \& Benjamin, 2017) “Flat-H Redundant Frangible Joint Design Evolution 2017”

2. (Diegelman, Hinkel, Benjamin, Rochon, \& Brown, 2016) “Flat-H Redundant Frangible Joint Evolution”

3. NESC-RP-13-00906 